\section{Nicolaas Bloembergen Arthur Schawlow}

\author{
S. D. Smith, Edinburgh \\ (Heriot Watt University)
}

The award of the Nobel Prize to both N. Bloembergen of Harvard University and A. Schawlow of Stanford University has given great pleasure to the Quantum Electronics Division of the European Physical Society.

Both Nicolaas Bloembergen and Arthur Schawlow had been intimately concerned with the early basic physics of the laser during the latter part of the 1950's and although Townes, Basov and Prokhorov were the first to be honoured by the Nobel Committee in 1964, Bloembergen and Schawlow's subsequent work on scientific applications of lasers has now led to a most satisfactory justice in the business of recognition of outstanding scientists.

Apochryphal stories abound over these matters: Mrs. Bloembergen is reputed to have asked Nicolaas on the occasion of a presentation to Charles Townes, in which Mrs. Townes received a ruby in recognition of the appropriate laser action, "That's nice. What would I have got if you had been successful?" Nico answered immediately, "My laser was made from cyanide." Art Schawlow's counterpart is his reported remark that he is pleased to have been awarded the Nobel Prize so that he will no longer have to keep explaining to people that he hadn't got it.

Nicolaas Bloembergen was born in Dordrecht in the Netherlands. He received his early degrees in 1941 and 1943 from the University of Utrecht and his Ph.D. in 1948 from the University of Leiden. His Ph.D. thesis research on nuclear magnetic resonance was performed at Harvard University in collaboration with Edward Purcell (himself a Nobel Laureate) and Robert Pound. After a short stay back at Leiden he settled at Harvard in 1949, becoming a naturalised US citizen in 1958. Since 1974 he has been Rumford Professor of Physics. His prolific research output includes over 200 papers in scientific journals and two well-known books. As with his co-Laureate his impact on optics owed something to having a foot in microwave physics, as exemplified by his book on Nuclear Magnetic Relaxation.

In the late 1950's, like Schawlow, he wrote speculative papers about laser action. Bloembergen's work was distinguished by including the effects of relaxation processes that unavoidably empty lower lasing levels, a vital feature absent from the papers of other authors at the time.

It is his contributions to the subject of nonlinear optics, however, for which Nico, as he is often known, is most famous (see inset). His papers on interactions between
Nonlinear optics is the generic title that describes what happens to materia when irradiated with the large intensities available in laser beams. Previously the dipole moment induced by the incident light wave was considered to be adequately described by one constant proportional to the amplitude of the electric field. However, laser fields comparable with inter-atomic fields can be readily obtained, so that the induced polarisation expanded in powers of the field can have significant values - up to at least the fifth order. In these circumstances, light frequencies can be mixed, and a rich variety of new nonlinear effects have been discovered. following 20 years that had not been anticipated. Thus the first observation of harmonic generation by Franken and colleagues found itself with a readymade framework for future developments. Bloembergen's book, Nonlinear Optics, although quite difficult to read, is an alltime classic. Characteristically he exploits density matrix notation to describe the quantum mechanics of nonlinear susceptibilities. This formalism has the virtue that it not only describes the rate of a quantum mechanical transition between two states, but it is also readily adapted to include population changes, and provides as well an averaging technique to describe scattering and relaxation processes in gases, fluids and solids. The framework developed was quite general and has been extensively used to describe all forms of nonlinear optical interactions.

Later contributions have included explanations of multiple photon dissociation of molecules, in particular the use of concepts of a quasi-continuum and a heat bath, and, more recently, the effects of relaxation processes in causing otherwise forbidden nonlinear processes in atomic vapours to be allowed. Nicolaas Bloembergen is universally regarded as the father figure of the subject of nonlinear optics.

Arthur Schawlow, an American born in Mt. Vernon, N.Y., was launched on his career in Canada at the University of

Nicolaas Bloembergen (left) of Harvard and Arthur Schawlow (right) of Stanford.

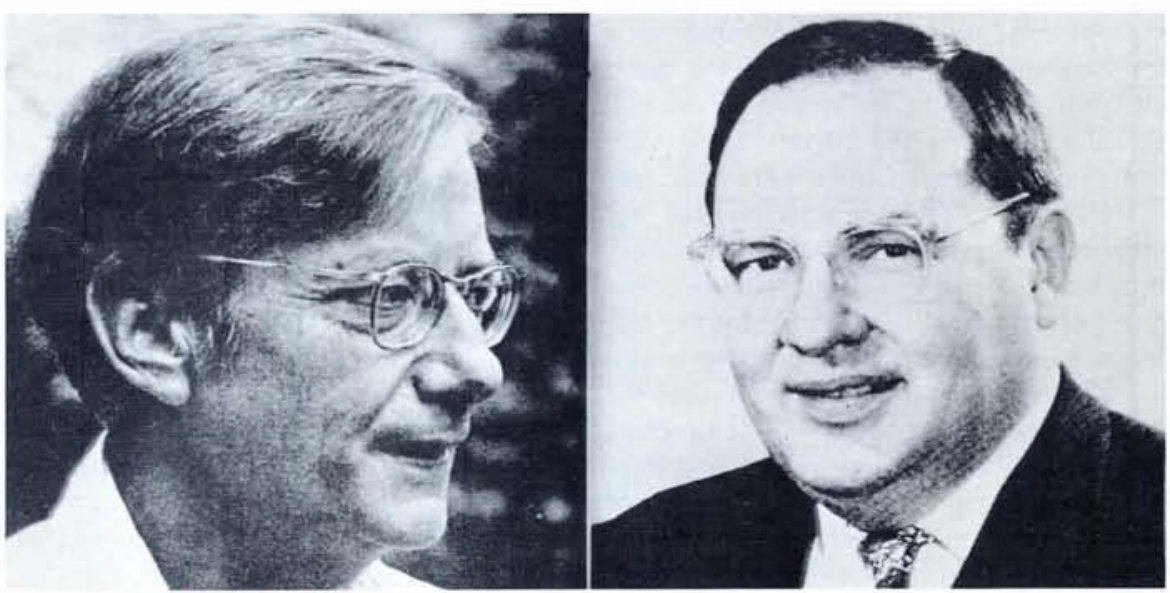


fine structure. The elimination of Doppler broadening was achieved by the method of saturation spectroscopy in which a laser beam is split into two unequal beams that cross in opposite directions the cell containing the atoms. Saturation spectroscopy itself was known from the pre-laser experiments of Purcell and Pound as early as 1948 and first achieved with a laser by Bennett, Lamb and MacFarlane. The strong beam 'bleaches' the absorption, which is detected via the intensity variations in the weak probe beam when the laser is tuned close to the atomic frequency, both light waves interacting with the same atoms, i.e. those with vanishing axial velocity. In Schawlow's work a narrow band laser whose frequency can be tuned continuous$l y$ across the spectral region of interest was employed. Dye lasers pumped by a nitrogen laser were brought to a high technical standard and first used to observe the hyperfine splitting of the $3^{2} \mathrm{~S}_{1 / 2}$ and $3^{2} \mathrm{P}_{1 / 2}$ states of sodium. Hydrogen, however, has always attracted attention because its simplicity allows accurate comparison with theory. Thus despite the large Doppler broadening $-6 \mathrm{GHz}$ at room temperature - resolution of the red Balmer line $\mathrm{H}_{\alpha}$ has been much sought after. Using the wide capability of the tunable dye laser, Schawlow and his group succeeded in resolving this structure and directly measuring the Lamb shift. Such measurements are the basis for precision determination of the Rydberg constant, one of the cornerstones of the evaluation of fundamental constants in physics.

These are just some examples of the outstanding work of two popular Nobel Prizemen of 1981 who will be honoured by the European Physical Society Quantum Electronics Division at a special reception at the 12th International Quantum Electronics Conference in Munich on 24 June 1982.

\section{Teaching Abroad}

Dr. Bengt Sandell of the University of Linköping has been invited by the Faculty of Arts and Sciences of Bogaziçi University, Istanbul to spend the spring semester as a Visiting Lecturer under the auspices of the Teaching Abroad Scheme of the European Physical Society. Sandell will be demonstrating his own particular skills in devising new experiments for teaching purposes and will be participating in the general work of the Faculty. This is the first result of the efforts that have been made by the Physics Education Committee of EPS to encourage the movement of teaching staff (as against research staff) at the university level.

\section{2nd General Conference of the Condensed Matter Division of the European Physical Society}

This conference will be a continuation of the general CMD Conferences started in 1980 in Antwerp. The sessions will cover the whole field of condensed matter physics with contributed papers and a large number of invited talks. Sixty invited speakers, many of them grouped into symposia, will cover the whole spectrum of solid state physics.

Chairman: Prof. V. Heine (Cambridge)

The Board of Governors of Eindhoven University of Technology announces an opening in the Telecommunications Division of the DEPARTMENT OF ELECTRICAL ENGINEERING for a

\section{professor (part-time) of optical-fibre communications,}

\author{
(male/female) \\ in particular, \\ glass-fibre technology and integrated optics.
}

Field The Telecommunications Division gives lectures and conducts research on electromagnetic communication channels and systems, notably for satellite communications and optical-fibre communications. The present activities in the latter field concentrate on optical transmission, detection, and system design.

Duties The succesful candidate is expected to give a lecture series dealing with the technology of optical fibres lincluding manufacturing, characterization and control, integration, and splicing of cables) and integrated optics (including the development of novel or improved components and their measurement). In addition, the professor is expected to assume responsibility for research in this area, and give direction to graduate students and to any postgraduate investigators pursuing a Ph.D. degree in one of these subjects.

Applicants should have adequate experience in the field and have an ability for teaching to which they should be prepared to devote one day per week. The appointment will be for three years, with possibility of renewal.

Applications or suggestions for suitable candidates are invited in confidence to the

Board of the Department of Electrical Engineering, Eindhoven University of Technology,

P. O. Box 513, $5600 \mathrm{MB}$ Eindhoven, the Netherlands.

These should arrive before 1 April 1982.

Further information may be obtained from prof.ir. J. van der Plaats, Tel. (40) 473451. 